\title{
Le jeu vidéo à Montréal : une négociation entre création et production
}

\section{Yann Pineault}

\section{(2) OpenEdition}

\section{Journals}

Édition électronique

URL : http://journals.openedition.org/sdj/494

DOI : $10.4000 /$ sdj. 494

ISSN : 2269-2657

\section{Éditeur}

Laboratoire EXPERICE - Centre de Recherche Interuniversitaire Expérience Ressources Culturelles Education

\section{Référence électronique}

Yann Pineault, «Le jeu vidéo à Montréal : une négociation entre création et production », Sciences du jeu [En ligne], 4 | 2015, mis en ligne le 04 octobre 2015, consulté le 12 avril 2021. URL : http:// journals.openedition.org/sdj/494; DOI : https://doi.org/10.4000/sdj.494

Ce document a été généré automatiquement le 12 avril 2021

\section{(c) (i) (9)}

La revue Sciences du jeu est mise à disposition selon les termes de la Licence Creative Commons Attribution - Pas d'Utilisation Commerciale - Pas de Modification 4.0 International. 


\title{
Le jeu vidéo à Montréal : une négociation entre création et production
}

\author{
Yann Pineault
}

1 Autour d'une table sont assis les principaux décideurs créatifs d'un projet de jeu vidéo, qui s'apprêtent à discuter d'armement. Une première proposition vient de l'artiste modélisateur en chef : celui-ci propose une sélection d'armes finement modelées et dérivées d'un même modèle de base dont les différentes parties seraient interchangeables, de façon à pouvoir modéliser plus d'armes, plus rapidement. Ces motivations vont de pair avec celles du designer en chef, spécialiste de l'expérience du joueur, souhaitant que ce dernier ait un choix d'armes qui semblent aussi distinctes les unes des autres, afin d'éviter un sentiment de répétition. Le directeur artistique, celui qui définit la ligne créative directrice, est sceptique face à l'utilisation d'un modèle interchangeable et croit que l'on devrait plutôt faire moins d'armes, mais qu'elles soient toutes singulièrement différentes.

2 La situation se corse quand l'animateur entre en jeu : il faut créer des repères afin de positionner les mains des personnages sur les armes. La diversité vient donc complexifier la tâche des animateurs. Les discussions continuent alors que l'artiste modélisateur et l'animateur attendent visiblement des directives claires pour diriger l'exécution technique; le designer en chef et le directeur artistiques ne sont pas d'accord sur la façon de procéder. C'est à ce moment que le producteur, gestionnaire du projet qui est resté silencieux jusqu'à maintenant, vient rappeler les modalités du contrat qui stipulent qu'un nombre donné d'armes doit être livré avec un seul style. Cet argument viendra clore le débat de sorte que seule une exploration visuelle sera permise, sans pour autant varier les mécaniques de jeu.

Une telle situation, très courante au sein du studio, met de l'avant une tension entre un espace réglé, celui de la production, et un espace d'exploration, celui de la création. Cet article s'intéressera précisément à cette opposition entre production et création par l'entremise de témoignages critiques de différents développeurs de jeux. Le point de 
vue des développeurs sera ici central puisqu'ils sont des agents importants dans l'évolution du jeu vidéo; leurs témoignages démontreront qu'ils voient l'innovation dans le jeu triple $\mathrm{A}^{1}$ comme étant stagnante ou du moins unilinéaire dans son évolution. Plus encore, nous tenterons de démontrer la façon dont se définit l'identité des développeurs-joueurs vis-à-vis de leur travail, tout en prenant en compte cet état de fait. Après avoir exposé les dimensions productives et créatives du développement d'un jeu, nous proposerons quelques réflexions sur le terrain de jeu qu'est l'industrie montréalaise. Celle-ci est encore jeune et nous verrons que la façon dont elle se structure a des effets importants sur la nature du jeu vidéo qui se situe entre l'objet d'art et le produit de masse. Ces pistes permettront de mieux comprendre les débats entourant l'innovation du médium ${ }^{2}$ dans un contexte local, tout en prenant en compte le fait que ce dernier évolue dans une réalité globalisée. Le cas de Montréal reflète bien le statut du médium à ce jour puisqu'on la considère aujourd'hui comme un lieu de production vidéoludique exemplaire sur la scène mondiale.

\section{Méthodologie}

Le présent article puise ses ressources dans une étude de terrain qui fut réalisée entre les mois de janvier et mai 2014. À l'origine, les données ont été collectées pour la réalisation d'un mémoire de maitrise en anthropologie (Pineault, 2014). La recherche, de nature ethnographique, avait pour but de connaitre les motifs et visions des développeurs de jeux vidéo face au travail de création et au produit lui-même dans un studio de production montréalais.

5 Comme nous l'avons souligné dans ce mémoire, il est désormais nécessaire de prendre en compte le point de vue des développeurs de jeux vidéo afin de mieux comprendre le médium à travers sa création et sa production. Non seulement le recours aux informateurs est central en anthropologie, mais il permet de voir l'objet vidéoludique sous un autre angle, que l'on voudrait ici dissocier d'une approche formaliste. L'un des risques de l'utilisation d'une approche formaliste est que : «[...] son approche du jeu comme objet a pu tendre vers une acception essentialiste de ses propriétés, et conduire à une perception du jeu comme activité qui confine parfois au déterminisme technique " (Zabban, 2012, p. 167). Notons toutefois un intérêt grandissant pour le point de vue des game designers (Hock-Koon, 2011; Chiapello, 2014) dans la mesure où ils partagent un langage commun avec le chercheur. Par contre, il semble nécessaire de préciser qu'une attention doit être portée sur l'ensemble des développeurs dont les perspectives peuvent diverger. Par exemple, le point de vue des producteurs, ces développeurs veillant à la bonne réalisation du projet, est essentiel puisqu'ils ont un poids important dans la création d'un jeu vidéo.

6 Les données ont été collectées à travers deux techniques : l'observation participante et la réalisation d'entrevues ethnographiques. La première technique a permis de saisir la dynamique d'un studio de production vidéoludique en profitant des aléas du milieu de travail (shadowing, rencontres fortuites, réunions formelles et informelles, atmosphère générale, etc.). Nous avons participé activement en tant qu'observateur à la vie au quotidien dans un studio de production vidéoludique afin d'en comprendre la culture organisationnelle. Ces observations allaient de pair avec la réalisation d'entrevues semi-dirigées puisqu'elles nous fournissaient un matériau de base sur lequel construire nos questions, qui furent relatives aux connaissances déjà acquises sur l'individu ou son 
milieu de travail. ${ }^{3}$ Les individus interviewés ont d'abord été choisis en fonction de leur volonté de participer à l'étude, mais aussi de façon à obtenir des témoignages représentatifs des différents métiers impliqués dans le projet observé (designers, artistes modeleurs et animateurs, programmeurs, directeur artistique, producteur et assurance de la qualité).

7 Les entrevues étaient de nature ethnographique puisque notre contact avec les informateurs ne se limitait pas au contexte de l'entrevue, mais se caractérisaient aussi par une volonté de créer un lien de confiance avec ces derniers. Les témoignages obtenus reposaient également sur une approche phénoménologique, afin de rendre compte de réalités individuelles telles qu'elles se présentent par la voix de nos informateurs (Paillé \& Mucchielli, 2012). Par ailleurs, d'autres entrevues semi-dirigées ont été réalisées avec des acteurs gravitant autour de l'industrie du jeu vidéo: enseignants, chercheurs et consultant ont été interrogés dans le but d'obtenir différentes visions de l'industrie et permettant, par le fait même, une plus grande richesse discursive au sens foucaldien du terme (Foucault, 1971). Cette approche fut centrale dans notre démarche puisque relever les disparités discursives permet de mettre à jour la teneur des idées qui circulent et s'opposent dans un espace social donné. C'est le propre d'une approche multisite (Marcus, 2002), puisqu'elle tente de rendre compte d'une réalité ethnographique dépassant une réalité locale et qui serait confinée dans un ethnos particulier. Enfin, nous avons effectué une analyse phénoménologique des données afin de cerner l'expérience vécue des développeurs. Ces constats ont par la suite été regroupés à l'aide de catégories conceptualisantes afin d'en faire une analyse systématique qui nous a permis d'observer cette tension entre production et création.

Nos observations ont été effectuées lors de la préproduction d'un jeu vidéo dans une équipe d'environ quarante personnes au sein d'un studio montréalais représentant un échantillon de développeurs expérimentés. Si le point de vue d'une main d'œuvre junior y est moins représenté, nous avons eu la chance d'obtenir le témoignage de développeurs qui ont vu l'industrie et le médium se transformer. Enfin, nous avons choisi de taire l'identité des informateurs en leur attribuant des noms fictifs de façon à respecter la confidentialité dans un milieu industriel où les informations peuvent rapidement devenir sensibles.

\section{La production comme espace réglé}

9 Si un besoin de liberté et d'épanouissement se fait ressentir du côté des développeurs dans la production d'un jeu vidéo, l'espace réglé de la production ne cesse de rappeler qu'elle vient structurer une activité qui autrement serait vue comme futile. C'est ce regard que nous tenterons ici de comprendre, non pas dans le but de le critiquer, mais de l'incruster dans une réalité aux dimensions multiples.

Selon nos informateurs, les contraintes liées à la production d'un jeu triple A sont élevées : la nature d'un tel projet peut être rapprochée de celle de la course, où la pole position semble être la seule option viable :

[La compétition] est très féroce : il y a beaucoup d'appelés et il y a peu d'élus. De plus en plus on voit qu'il y a concentration du marché auprès de quelques jeux qui sortent par année puis qui font la majeure partie du chiffre d'affaires. Oui, la compétition est extrêmement vive. (Gaétan, chef de studio) 
11 Il s'agit d'une compétition où seulement quelques grands jeux rafleront la majeure partie des revenus et, vu les budgets faramineux investis dans ces productions, la marge d'erreur devient très mince. Qui dit course, dit direction univoque et celle-ci suit la trajectoire telle qu'elle fut présentée par les auteurs de Digital Play (Kline, DyerWitheford \& De Peuter, 2003) dans leur revue historique critique de l'évolution du médium vidéoludique: soit une valorisation technologique axée sur l'immersion et le réalisme graphique. La majorité des développeurs interrogés avaient des discours très critiques à l'endroit de cette forme d'innovation qu'ils jugent unilinéaire. Ces derniers croient que l'innovation vidéoludique passe avant tout par la diversification des expériences de jeu, mais un retour constant est fait vers ce qu'on pourrait qualifier de système de contraintes. Bien que le discours technologique soit surtout l'apanage de la production, les développeurs sont conscients des impératifs technologiques venant structurer le développement d'un jeu AAA. Selon Luke, directeur créatif :

Personne ne sait comment sortir de cette "chasse au dragon" que sont les graphiques et la technologie et ce n'est pas comme pour le matériel, les coûts ne diminuent pas au même rythme que les technologies évoluent (notre traduction). ${ }^{4}$

Pour une grande compagnie, créer un jeu est d'abord un investissement et qui dit investissement dit retour sur investissement. Les projets mis en place sont ceux que l'on juge les plus fertiles en utilisant comme indicateur le marché et les ventes des dernières productions vidéoludiques. Les projets sont ainsi l'objet d'une commande qui vient des investisseurs moins que d'une idée ingénieuse émergeant d'esprits créatifs :

Ce que j'aime pas dans cette industrie, je te dirais [que] c'est les attentes. C'est de se dire: on est une grande compagnie, voilà notre catalogue de jeux; or, voilà le catalogue de jeux de la compagnie concurrente, ils ont ça, on l'a pas, faisons-le. Ce que je veux dire c'est que la plupart des jeux maintenant deviennent des commandes, c'est-à-dire, tiens il nous faut un nouveau jeu $\mathrm{X}$, faisons un nouveau jeu $\mathrm{X}$. À la base il y a pas vraiment eu quelqu'un au niveau créatif qui a dit : «ça serait intéressant ». (Adam, directeur du design de niveaux).

La conception du jeu se fait par un choix de caractéristiques que l'on doit inclure dans le jeu de façon à susciter l'engouement des joueurs. Une approche procédurale de la sorte a un effet sur la diversité des expériences vidéoludiques offertes par les grandes compagnies puisqu'on crée un idéal-type du joueur à qui l'on veut adresser ses produits. Cette façon de faire entre en conflit avec la volonté de créer des développeurs, et même si une exploration est par la suite nécessaire autour de ces caractéristiques, il n'en demeure pas moins que l'expérience ludique est formatée par ces choix préliminaires.

Ted $\mathrm{F}$. Tschang utilise le concept de rationalisation pour décrire ce phénomène où :

Les produits créatifs tels que le film et le jeu vidéo sont de plus en plus complexes et coûteux à développer, ce qui mène à la rationalisation de la production ou du processus de développement de produits. Dans cet article, la rationalisation est définie comme étant l'accent mis sur les intérêts d'affaires ou les processus de production orientés vers la productivité, habituellement au détriment de la créativité (Tschang, 2007, p. 989, notre traduction).

14 Cette rationalisation, bien que nécessaire, se fait au dépend de la créativité et il s'agit d'un aspect qui nous a été maintes fois souligné. L'usage de l'expression «travail de commande » par certains de nos informateurs est très évocateur de la hiérarchie qui subsiste dans un milieu de travail qui se veut pourtant très horizontal. Sam par exemple, designer, n'hésite pas à affirmer que son travail est toujours l'objet d'une commande venant de quelqu'un au-dessus de lui : 
Et que tu sois junior, t'auras toujours un gars qui va vérifier ce que tu fais, quand t'es senior, t'as toujours un lead au-dessus de toi, quand t'es lead, t'as toujours un directeur au-dessus de toi, quand t'es directeur, t'as un directeur créatif au-dessus de toi, quand t'es directeur créatif t'as un directeur de production ou des stakeholders, donc t'es jamais libre de faire ce que tu veux et je pense que ça, ça peut être très difficile et de ressentir le poids de la hiérarchie et des décisions qui sont prises par d'autres, je pense que ça peut être très très dur pour certaines personnes.

Ce genre de commentaires critiques survient dans la mesure où beaucoup de développeurs voient leur travail sous l'ordre de la technicité, moins que de la créativité. Adam par exemple se considère comme un "terminal créatif » plutôt qu'un créateur. Le terme est fort et les répercussions identitaires le sont tout autant dans la mesure où l'on se voit comme une main d'œuvre créative pour l'industrie. D'autres développeurs ont toutefois moins d'ambitions créatives personnelles et voient ce genre de réactions comme étant le fait de juniors dépassés par leurs ambitions. C'est le cas de Bruce, directeur artistique, affirmant que :

Ce sont surtout les juniors qui ont de la difficulté avec cela. Ils ont tendance à être plus attachés à leur travail et penser à une œuvre singulière et tout ça, et sont plutôt intéressés à créer des pièces de portfolio. Mais je crois que n'importe qui avec un rôle plus senior qui est dans l'industrie depuis un certain temps, réalise que personne ne verra une idée si elle n'aboutit pas à un jeu. Il est de notre intérêt de faire en sorte que le jeu soit expédié et fonctionne, donc mettons l'accent sur le produit et non pas sur les caractéristiques individuelles de ce dernier.

Or, nous verrons plus loin que certains acteurs critiquent cette tendance qu'a l'industrie à rabrouer les esprits plus créatifs en les confinant à leurs fonctions techniques. Qui plus est, l'industrie montréalaise du jeu vidéo a pris beaucoup d'ampleur en très peu de temps (Technocompétences, 2013) et ce faisant, la taille des équipes par projet a beaucoup augmenté. Suite à l'implantation d'Ubisoft, dont le nombre d'employés a été multiplié de façon exponentielle, plusieurs grands studios internationaux tels que Eidos, EA et Warner Bros Games se sont établis à Montréal. Si l'industrie employait environ 1200 travailleurs en 2002, elle en comptait près de 9000 en 2012. De plus grandes équipes contribuent à la spécialisation et à la valorisation d'une expertise de plus en plus pointue. Hélène, productrice, croit que :

Plus les équipes sont grosses, les gens sont de moins en moins multidisciplinaires, ce sont de moins en moins des généralistes, ce qui veut dire que tu te retrouves très rapidement avec » oui, mais tel problème ne peut être fixé que par tel autre gars » et personne ne veut aller toucher à ses affaires et lui ne veut pas toucher à celles des autres.

17 Cette spécialisation va de pair avec la professionnalisation des métiers du jeu vidéo qui s'est progressivement effectuée et qui a transformé l'industrie montréalaise. À ses débuts, elle accueillait des professionnels dont les compétences pouvaient être importées puis transformées pour faire du jeu vidéo, mais aussi des individus créatifs qui ont monté les échelons en passant de testeurs à designers, par exemple. Ces parcours sont cependant devenus rarissimes alors qu'un engouement s'est créé chez ces jeunes femmes et hommes qui pouvaient désormais aspirer à faire un travail de leur passion. Des formations spécifiques ont été mises en place autour des métiers du jeu vidéo afin de créer une main d'œuvre plus compétente, mais surtout prête à travailler dans l'industrie. ${ }^{5}$ La plupart de ces formations sont directement axées sur les besoins de l'industrie et ce faisant, des professeurs comme Roger, enseignant en modélisation 3D, 
croient que les étudiants forment d'excellents techniciens sans pour autant développer leur esprit critique. Il précise :

Ce qui manque en ce moment peut-être, c'est le pont entre l'industrie puis l'académique. Alors qu'avant l'académique c'était juste une machine à sortir des étudiants ; ce que nous on aimerait, c'est que l'industrie revienne chez nous et dise : " on a un projet, on aimerait ça travailler sur l'Oculus Rift ${ }^{6}$ pour du casual gaming ». Qu'ils viennent nous voir et nous demandent si on a une équipe qui peut travailler sur ce projet-là.

Un discours critique s'est construit au sein même de l'industrie et se veut une réponse face aux contraintes de productions d'un jeu triple A ne laissant qu'un faible espace créatif. Le travail dans un studio de jeu s'affiche comme un ordre négocié dans lequel la production tente réellement de donner une marge de manœuvre aux développeurs, mais où les règles s'avèrent rabroueuses dans leur façon d'encadrer les pratiques créatives. Il convient de se demander si les attentes des développeurs peuvent se concilier aux modalités de production. Mais avant cela, nous nous concentrons sur cette visée créative et ce qu'elle évoque chez les développeurs.

\section{La création comme espace libre}

19 Le statut de développeur (de jeux vidéo comme de logiciels au sens large) s'est quelque peu transformé avec le temps. Les histoires de réussites de petites boîtes californiennes ne sont pas si loin derrière nous et ont contribué à créer une image prestigieuse de ces jeunes génies qui, à partir de rien (si ce n'est leur ténacité et leur ambition), ont réussi à mettre en place des programmes informatiques innovateurs. Ces succès ont leurs équivalents au niveau du jeu vidéo avec les Will Wright, Sid Meier ou Richard Garriott ${ }^{7}$ qui ont, chacun à leur façon, révolutionné le monde du jeu vidéo. Ces histoires créent un espace nostalgique avec ses propres marqueurs auquel plusieurs de mes informateurs ont fait référence puisqu'il offrait, selon eux, des conditions de création (ou d'innovation) idéales.

On l'a par exemple vu lors d'une présentation de Philippe Ulrich (2014), fondateur de la compagnie de jeu Cryo Interactive, au cours de l'édition 2014 du symposium annuel Histoire $d u j u^{8}$ : la création d'un jeu vidéo pour micro-ordinateur était basée sur les compétences des individus à utiliser, voire détourner les fonctions de l'outil informatique et à y attacher un design vidéoludique conséquent. Avant l'arrivée de façons de faire bien documentées et enseignées dans les centres de formation, les développeurs ne disposaient que de peu de moyens et devaient faire un usage judicieux des technologies disponibles (Kline, Dyer-Witheford et De Peuter, 2003, pp. 84-108); le succès était souvent inattendu.

21 Les développeurs avec qui nous avons pu échanger étaient majoritairement des vétérans et ont été témoins de l'évolution de l'industrie. Pour la plupart, ils et elles ont un regard nostalgique sur leur carrière et particulièrement sur leurs premiers projets qui se caractérisent par la passion, le manque d'organisation, et surtout un nombre incalculable d'heures de travail. C'est quelque chose dont Sam se rappelle avec nostalgie :

On vivait au travail, j'ai jamais revécu ça. Tout le monde faisait de l'overtime jusqu'à minuit, on revenait les fins de semaine. On venait la fin de semaine déjà rien qu'en préproduction, il y avait déjà quelqu'un, on commandait de la bouffe tous les soirs, c'était assez incroyable. Je ne dis pas que c'est comme ça qui faut faire des jeux, 
mais personnellement j'ai trouvé ça incroyable, c'est comme si on était une grosse bande de passionnés dans un immense garage. Je suis vraiment très nostalgique de cette période-là. modalités plus strictes.

Ce dernier est donc conscient que les choses ont changé, tout en donnant l'impression que quelque chose s'est perdu. Ces premiers projets sont perçus comme étant des espaces d'apprentissage, d'expérience, mais surtout de création; espaces que les développeurs semblent avoir de plus en plus de difficulté à retrouver dans les studios triple A tels qu'ils se sont actualisés. Il peut être difficile d'exprimer ses idées dans le contexte contemporain ; même si des moments sont désignés à cet effet dans le studio, il n'en demeure pas moins que la plupart de ces idées seront écartées pour revenir à des

En parallèle, nous avons croisé un discours récurrent valorisant le milieu du jeu indépendant, soit la production de jeux vidéo avec un budget très restreint, de petites équipes, sans grand éditeur et une liberté créative presque totale. Non seulement plusieurs des développeurs encourageaient le jeu indépendant, mais y voyaient un espace permettant l'innovation du médium vidéoludique, puisque les contraintes ne sont pas les mêmes qu'au niveau du triple A. Sam, encore une fois, voit définitivement son avenir dans le monde du jeu indépendant:

Personnellement, je trouve de plus en plus difficile d'exister en tant que créateur, je ne dirais pas créatif parce qu'il y a de la création dans mon métier au jour le jour, mais d'être capable de porter une idée et justement de prendre des risques en terme de mécaniques et même de thèmes et de ne pas faire l'objet d'un travail de commande, c'est quelque chose qui m'intéresse très très fortement. Et je vois mon futur dans la scène indépendante et de ne pas rester dans le triple $\mathrm{A}$ toute ma vie, parce qu'à un moment donné t'as envie de développer des choses pour toi, t'as des choses à dire personnellement qui ne sont pas les mêmes qu'une grosse entreprise.

Il ne s'agit pas seulement de création, mais d'être créateur au sens littéral, de pouvoir se reconnaitre dans le travail effectué. La reconnaissance du travail en tant que créateur est un thème qui est récurrent dans nos discussions. Lorsque les équipes peuvent compter plusieurs centaines voire milliers de développeurs, il devient difficile d'obtenir une reconnaissance directe, puisque celle-ci est habituellement rendue au directeur créatif. Le jeu indépendant est un modèle d'entreprise permettant l'expression créative individuelle tout en favorisant une révision de l'expérience ludique ; comme le souligne Adam :

Les jeux indépendants détruisent ce moule et en créent un et c'est ça qui intéresse les gens et c'est ça qu'on faisait à l'époque au tout début des jeux : on vendait un moule différent et on disait quelle espèce d'expérience et de rythme on va vendre. À l'époque des pong-like, on appelait ça un clone, c'était plus honnête que maintenant où l'on nomme cela une licence.

C'est une avenue qui favorise un retour aux sources, au sens où les développeurs tentent de différencier leurs jeux des autres productions en faisant un usage efficace des ressources qui sont, pour ainsi dire, limitées. Un parallèle peut-être tracé avec le témoignage d'Ulrich cité plus haut, mais aussi celle des premiers projets, où les développeurs font un retour nostalgique à cette façon dont on faisait autrefois les jeux vidéo qui se voulait innovante. C'est souvent ce qu'entendaient nos informateurs lorsqu'ils faisaient allusion à l'innovation dans l'industrie du jeu vidéo, soit un usage détourné des technologies disponibles aboutissant à de nouvelles formes de jouabilité.

Les développeurs de jeux indépendants ont conservé cette vision dans la création de jeux ; c'est une recette qui comporte son lot de succès et auxquels s'intéressent de plus 
en plus les médias. Pensons par exemple à l'histoire de Simon Darveau, ancien designer chez Ubisoft qui a cofondé Spearhead Games et qui voit le jeu indépendant comme le futur de l'industrie vidéoludique montréalaise, voire même du médium. ${ }^{10} \mathrm{Ce}$ secteur se voit aussi garant d'une relève ne voulant pas strictement effectuer un travail technique dans un milieu industriel stagnant et où les décisions concernant la création ne sont prises que par les développeurs expérimentés.

Cependant, force est de constater que peu de développeurs sont prêts à faire le saut, même s'ils valorisent beaucoup ce secteur. Les postes de développeurs d'expérience sont hautement rémunérés dans le milieu du triple $\mathrm{A}$ et passer du côté indépendant comporte un grand risque financier. ${ }^{11}$ Plus encore, la compétition n'en est pas moins vive du côté des petites productions ; des plateformes comme Steam, mise en place par la compagnie Valve Corporation, permettent la distribution de jeux sans l'intermédiaire d'un éditeur. Le défi est donc de se démarquer parmi une mare de produits qui ne cesse d'augmenter. Jordan, directeur technique, nous en dit un peu plus sur la segmentation des marchés vidéoludiques :

Dans les indy [pour indépendants], c'est difficile parce que ça coûte cher de faire un jeu pis après ça souvent t'as pas d'argent pour le marketing, donc là faut que tu aies des partenaires pour ça, donc je pense que c'est difficile de faire de l'argent. Sauf que tu peux être plus créatif, t'as moins de contraintes parce que dans le triple A t'as plein de contraintes. T'as ton publisher, ta compagnie, t'as beaucoup de monde qui travaille aussi, c'est des équipes de 100 à 2000 personnes. Les indy c'est beaucoup plus difficile; pour fonctionner, c'est des plus petites équipes. Y'a des jeux extraordinaires là-dedans, sauf qu'après ça, tu peux pas vendre un jeu indy à $80 \$$ comme un triple $\mathrm{A}$, ça ne vaut pas ça l'expérience, à mon avis, c'est pour ça que c'est un jeu indy pis qu'ils vendent ça 20-30 \$ sur Steam. Si tu fais un bon jeu, t'as des chances que ça marche, mais ça marchera jamais autant que le Call of Duty ou le Grand Theft Auto ${ }^{12}$, ça ne sera pas 10 millions de copies vendues.

Pis si tu vas dans le mobile, ${ }^{13}$ c'est encore pire je pense parce qu'il y en a vraiment beaucoup puis pour que ça marche, dans le top 25 mettons sur iOS [le système d'exploitation pour mobile développé par Apple], ben si tu veux être là c'est même pas $1 \%$ des jeux. Donc il faut que tu aies un bon jeu, mais après ça faut que tu aies des bonnes stratégies de marketing virales, etc. pour que ton jeu puisse se propager là.

Cette longue citation démontre bien qu'une segmentation du marché s'opère entre les différents modèles. Certains développeurs, comme Jordan, croient que cette diversification est saine, puisque les expériences offertes seront différentes. Il s'agirait d'un prix à payer pour être indépendant au niveau créatif et un tel propos vient renforcer la précédente tension qui s'opère entre l'espace contraint, mais réglé et l'espace libre et créatif.

29 Tous les développeurs interrogés s'entendent pour dire qu'ils aimeraient voir plus d'innovation dans le secteur triple A et, bien que certains envisagent de créer leur propre compagnie, la plupart entretiennent plutôt cet intérêt par nostalgie ou par fantasme, puisque peu d'entre eux seraient prêts à mettre de côté leur sécurité d'emploi.

Je te dirais que c'est un risque. Si j'avais pas une famille avec des enfants, peut-être. Ça demande quand même un certain investissement personnel et puis en même temps, tu peux réussir à avoir les subventions du gouvernement, j'ai plein d'amis qui ont fait ça et ça veut pas dire qu'ils font une fortune contrairement à ce qu'on croit. [...] Je ne sais pas si je suis prêt à prendre ce risque...C'est séduisant, mais... (Adam) 
Ces témoignages critiques soulignent une volonté de changement. Plus qu'une nostalgie, ces développeurs croient qu'il serait nécessaire de remettre cet esprit créatif à l'avant plan, esprit entourant la naissance de plusieurs projets qui ont laissé leurs marques dans l'univers vidéoludique. Suite à cette présentation, il convient de se questionner sur différentes avenues susceptibles de créer un terrain de jeu mieux adapté, où les règles ne prennent pas le dessus sur l'épanouissement, sans pour autant tomber dans une fantaisie incontrôlée, pour utiliser les termes de Caillois (1967).

\section{Réflexions autour du « terrain de jeu »}

31 Il est important de comprendre que la façon dont l'industrie montréalaise s'est structurée joue pour beaucoup dans sa façon de promouvoir le jeu vidéo, justifiant ainsi les témoignages présentés jusqu'à maintenant. Dans cette section, il convient d'effectuer une distanciation face aux discours présentés ci-dessus afin de comprendre comment ceux-ci sont ancrés dans une réalité socioéconomique plus large. Comme l'a démontré Laurent Simon dans sa thèse de doctorat consacrée à Ubisoft (Simon, 2002), les premiers balbutiements dans la multinationale française en sols montréalais se caractérisaient principalement par l'apprentissage. Douze années plus tard, notre étude a pu faire état d'une structuration sous plusieurs dimensions à travers les différents témoignages de développeurs et acteurs connexes (Pineault, 2014). Nous en avons fait état plus haut: les façons de faire se sont consolidées autour des centres de formation, créant ainsi une main d'œuvre adaptée, mais aussi du côté des développeurs expérimentés. Ces derniers ont raffiné leur expertise au fil du temps et c'est quelque chose qui se ressent dans le milieu de travail: l'ethnographie de Simon racontait l'histoire d'un savoir en train de se créer alors que la nôtre atteste d'une professionnalisation et d'une tension dans la façon de concevoir son rôle de développeur. Nous croyons que l'industrie montréalaise se situe dans une phase de transition, à l'instar de la scène vidéoludique mondiale, où l'on se demande si le jeu triple A pourra conserver son modèle d'affaires actuel avec l'arrivée de la prochaine génération de consoles (Rayna \& Striukova, 2014).

Économiquement, l'industrie montréalaise s'est surtout fait valoir par ses grandes entreprises; ce sont elles qui emploient la plus grande partie des développeurs et qui créent des jeux triple $A$, marché qui s'avère toujours le plus rentable en terme de revenus (Technocompétences, 2013; Entertainment Software Association of Canada [ESA], 2014). Plus encore, ce sont ces mêmes entreprises qui reçoivent la majeure partie des subventions et crédits d'impôt accordés par le gouvernement québécois en échange du nombre important de postes qui sont créés. ${ }^{14}$ Une équation du succès s'est donc mise en place autour de façons de faire qui se sont solidement ancrées et renforcées au fil des réussites québécoises en matière de jeux vidéo. Cependant, des développeurs comme Adam croient que cette même équation engendre un conservatisme au sein des grands studios, empêchant l'innovation du médium.

33 Roger, professeur en création 3D cité plus haut, jugeait l'innovation par sa progression unilinéaire ; celle-ci serait surtout axée sur l'aspect technologique en voulant sans cesse repousser les limites du photoréalisme. Dans un même ordre d'idées, Réal, propose un témoignage axé sur la condition des designers, domaine dans lequel il est formateur. Il croit que devant une recette qui a son lot de succès, mais aussi un modèle d'affaires 
contraignant, les façons de concevoir les jeux vidéo se cristallisent autour de concepts et d'expériences similaires :

C'est-à-dire que quand il faut performer, quand il faut fournir des prototypes rapidement, quand il faut valider une idée de façon définitive parce que le marketing attend de vendre l'idée le plus vite possible, c'est certain que cette pression-là nous permet pas d'entrer dans un grand chaos créatif. Cette pression-là nous emmène souvent à faire des choix rapides et les choix rapides vont souvent venir de ce qu'on connaît le mieux. Si on est que plongé dans le jeu vidéo, et bien on risque de reproduire ce que le jeu vidéo fait le mieux, c'est-à-dire des interactions souvent basées sur la dextérité, la rapidité et l'élimination d'agents dans le jeu.

Cette reproduction est d'abord causée par le financement même des projets: les investisseurs choisiront ou non de financer un projet en fonction de son succès potentiel, à la lueur des succès en vogue sur le marché. Le phénomène des clones (ou licences) fait en sorte que des modes vidéoludiques verront le jour: devant le succès d'un jeu innovateur, plusieurs entreprises tenteront leur chance en créant un jeu similaire. ${ }^{15}$ Sur le terrain, les développeurs sont aussi susceptibles de prendre part à cette reproduction: seuls les plus expérimentés proposeront des idées quant à la nature du jeu. Ces derniers sont à la base même de cette équation du succès dont je parlais ci-dessus et c'est surtout leur expertise qui est mise en avant dans la réalisation des projets vidéoludiques. Comme l'affirme Réal, ils auront souvent recours à ce qu'ils connaissent le mieux. Il devient donc difficile de faire valoir de nouvelles idées dans un milieu où la rationalisation a préséance, surtout pour les juniors, dont les postes sont axés sur la réalisation de tâches techniques. Toujours selon Réal :

On veut des gens qui vont pas trop faire bouger le cordage et qui vont laisser aller le bateau voguer sur un fleuve très tranquille et on aime mieux des gens souvent qui vont faire les tâches sans trop remettre en question les idées, les concepts et la façon de gérer. Est-ce qu'on engage les gens qui sont les meilleurs ou les plus dociles? Les gens qui ont un potentiel créatif et innovant ne vont jamais à mon avis faire de tors à un jeu ou à une production, en aucun cas. Il suffit seulement d'être capable de les gérer.

Du côté managérial, nous avons observé un véritable questionnement quant aux façons de stimuler la créativité tout en respectant les modalités de production. Les producteurs sont conscients, comme nous l'affirmait Hélène, que tous les développeurs ont «d'importants egos et savent comment faire un jeu » et c'est quelque chose qui devient difficile à gérer lorsqu'on doit respecter des échéanciers stricts. On retrouve donc un réel désir chez les producteurs et directeurs créatifs de créer des espaces d'idéation et de partage, mais ces idées avortent rapidement lorsqu'elles sont confrontées à un manque de communication entre les différents corps de métier ou tout simplement à des contrats de production trop déterminants. Devant l'échec de ces processus d'idéation, les développeurs sont portés à voir ces rencontres comme une perte de temps puisqu'ils y lancent des idées sans jamais qu'il leur soit donner suite. C'est pourquoi les développeurs font souvent allusion au milieu du jeu indépendant dans lequel il est possible d'agir pleinement en tant que créateur, vu la taille plus restreinte des équipes.

Force est de constater que l'industrie du jeu vidéo, en particulier à Montréal, est très jeune et qu'une «granularisation» (pour utiliser l'expression de Gaétan, cité plus haut) s'effectue progressivement au niveau des différents studios. En ce sens, la littérature et la presse utilisent de plus en plus la métaphore de l'écosystème pour caractériser l'entrepreneuriat vidéoludique montréalais. ${ }^{16}$ Le nombre de grandes entreprises stagne 
alors que les plus petites, œuvrant notamment dans les services connexes (son, assurance qualité, etc.), le jeu mobile et le jeu indépendant se multiplient (ESA, 2011, 2013 ; Alliance numérique, 2014). Si les développeurs font si souvent allusion au jeu indépendant, c'est que les histoires de succès se multiplient, notamment en sol montréalais et que c'est dans un espace moins contraignant que surviennent les innovations vidéoludiques.

Je ne pense pas que de façon générale l'innovation vienne du triple A, ça coûte trop cher. Tu peux pas prendre de risque et plus ça coûte cher, moins tu prends de risques. Donc plus tu vas vers des valeurs sures, mais plus aussi en tant que joueur ça dévalue un petit peu l'expérience. [...] Je pense qu'il y a de la place pour tout le monde [sur la scène vidéoludique], mais qu'il va y avoir de moins en moins de gros joueurs au niveau du triple A. (Hélène )

Nous ne prétendons pas faire état de la scène vidéoludique mondiale, mais il semble qu'un phénomène d'engouement se soit créé autour des plateformes de distribution comme Steam ou Kickstarter, site de sociofinancement où le public est invité à investir de petites sommes dans des projets nécessitant un financement. Face à de tels succès, ces développeurs répondent par une nostalgie créative et aspirent à rejoindre les rangs de ceux et celles qui vivent comme créateurs. Ici se pose la question de la valorisation de ces plus petites productions, au niveau économique et par extension, au niveau social. Le gouvernement québécois finance l'industrie du jeu vidéo et plus largement du «multimédia » sur la base du nombre d'emplois de qualité créés dans un domaine de pointe (Pineault, $2014: 60-82$ ). Les plus petits studios ne répondent cependant pas au critère de l'employabilité et tentent de faire entendre leur voix, ne profitant pas de la même valorisation gouvernementale qu'au niveau du triple A (Ouimet, 2013).

Selon Gaétan, l'industrie est encore "adolescente» et il est difficile de faire des prévisions face à l'évolution de la scène montréalaise et du médium lui-même. Cela dépend de la façon dont l'industrie se sédimentera et, plus important encore, de l'évolution du joueur-type et de ses préférences en terme d'expérience vidéoludique. Une question se pose à savoir si le jeu indépendant évoluera de la même façon que le cinéma indépendant, soit s'il deviendra l'apanage d'une niche de joueurs bien définie ou s'il deviendra un modèle d'innovation économiquement viable. À ce jour, le jeu indépendant agit à titre de catalyseur d'innovations vidéoludiques et ce sont plutôt les grandes compagnies qui emboîtent le pas en réutilisant les licences ou en achetant tout simplement les entreprises et leurs concepts. Réal croit qu'il s'agit d'un choix de société ; si une certaine fierté s'est créée autour du jeu vidéo (Pineault, 2014 :60-71), la suite dépendra du statut qui sera donné au produit :

[...] c'est trop jeune la discipline, on n'est pas encore rendu au stade où on considère vraiment le jeu vidéo comme un art, à part entière. On n'est pas encore rendu au stade où on va nécessairement, en tant que collectivité, accepter que l'on donne des sommes intéressantes à des gens pour créer à partir de ce médium-là. Pour l'instant on accepte, puis parfois on accepte trop, je considère qu'on exagère un peu avec l'industrie du jeu vidéo, mais on accepte surtout qu'on donne de l'argent pour faire fonctionner l'industrie qui embauche des travailleurs.

Un équilibre reste donc à créer entre la vision du jeu vidéo comme objet d'art (partagée par les développeurs indépendants) et celle du jeu vidéo comme produit de masse (partagée par les dirigeants des grandes entreprises). Les répercussions économiques de cette industrie ont maintes fois été mises en avant (Alliance numérique, 2014), ${ }^{17}$ mais il demeure qu'on en connait assez mal les retombées qualitatives à ce jour. Comment perçoit-on socialement le jeu vidéo suite à son implantation au Québec? Quel statut 
attribue-t-on au jeu vidéo produit localement? Une prise en considération de ces questions permettrait d'évaluer si une véritable culture du jeu s'est construite en sol montréalais, au-delà d'une industrie lucrative. C'est par la création d'une culture du jeu solidement ancrée que passera l'innovation de ce dernier, moins que par la stricte formation d'une main-d'œuvre qualifiée, qui n'est en fait qu'un aspect de cette culture.

Une piste à creuser se trouve dans les communautés, qui ont notamment été étudiées par le groupe de recherche Mosaic (Cohendet, Grandadam \& Simon 2010, Grandadam, Cohendet \& Simon 2013, Simon \& Cohendet 2007). Ces auteurs soulignent l'importance d'étudier les communautés et les façons dont elles sont liées entre elles afin de comprendre la nature de la ville créative, puisqu'un développeur n'appartient rarement qu'à une seule communauté. Pensons aux organisations, espaces et lieux de rencontres, ordres professionnels ou au jeu lui-même, pour ne nommer que ceux-ci. Les auteurs croient que Montréal possède des éléments structuraux (au sens structuraliste du terme) permettant l'émergence d'idées et de comportements créatifs. Paul, chef de studio et ex-consultant pour l'industrie du jeu vidéo, affirme que :

[...] les développeurs ont réellement un sens d'appartenance à une communauté plus large qu'est l'industrie du jeu et il y a une certaine affiliation, une association intangible à cette communauté qui dans certains cas va au-delà de leur affiliation à leur employeur actuel. Je pense que pour certaines personnes : «je suis avant tout un créateur de jeux et ensuite un programmeur pour une compagnie $\mathrm{X} »$. Je ne crois pas que tous les développeurs partagent cet état d'esprit, mais il y en a certainement beaucoup. La passion t'emmène vers ces communautés et espaces où tu veux partager, apprendre et sentir que tu appartiens à cette communauté.

Ces espaces, complémentaires aux milieux de production que sont les studios, sont importants à considérer dans la mesure où ils représentent des milieux de partage axés sur le médium lui-même. Le studio n'est pas en mesure de fournir ces espaces de réflexion puisque les grandes entreprises œuvrant dans le milieu du triple A sont directement en compétition. Plus encore, les développeurs sont majoritairement des joueurs eux-mêmes et tous n'ont pas la chance de mettre de l'avant leurs idéaux, non seulement en terme créatif, mais aussi en terme de préférences vidéoludiques.

Si les précédents auteurs ont bien démontré la façon dont se compose la ville créative, notamment au travers d'un modèle exprimant la circulation des idées et connaissances en termes de création, il n'en demeure pas moins que d'autres études de terrain seront nécessaires afin de saisir la nature des différentes communautés habitant la scène vidéoludique montréalaise. Une plus grande connaissance de ces communautés permettrait, entre autres, de créer des chemins de traverse. En ce sens, des liens sont encore à tisser entre les entreprises, les milieux de formation techniques, les communautés de développeurs-joueurs et les universités qui voient tous l'objet vidéoludique de façons différentes. L'évolution du jeu vidéo en termes d'innovations, devra donc passer par la mixité des différentes perspectives, mais aussi par de multiples communautés de façon à enrayer le conservatisme et mettre de l'avant des options économiquement viables pour faire évoluer le médium.

\section{Conclusion}

La mise en situation au début de l'article nous donnait déjà quelques pistes : créer un jeu vidéo triple A relève d'un ordre en constante négociation opposant les visées créatives et les modalités de production. Les témoignages de développeurs sont venus 
souligner la rationalisation qui s'opère dans un grand studio montréalais, mais surtout, ils ont mis en avant une identité de créateur s'épanouissant difficilement dans leur milieu de travail. Cette identité, faisant un retour nostalgique (voire romantique selon Gaétan) sur les anciennes productions, soulève un questionnement important quant à l'évolution à venir de l'industrie et du médium. L'industrie du jeu vidéo montréalaise est dans une phase " adolescente "; les pions bougent rapidement sur l'échiquier, alors que les jeux en ligne et mobiles acquièrent une part grandissante du marché.

Plusieurs scénarios sont envisageables, mais il semble que le statut attribué au jeu vidéo, et par extension à celui de développeur, sera déterminant dans l'évolution du médium en sols montréalais. Plus encore, ce statut saura faire la différence entre une vision de Montréal comme ville créatrice de produits innovants et Montréal comme milieu disposant d'une main-d'œuvre qualifiée et de crédits d'impôts avantageux pour les capitaux étrangers. L'une n'exclut pas l'autre, mais tous deux attestent de finalités différentes, voire opposées : la première est susceptible de faire progresser le jeu vidéo comme objet d'art par l'expérimentation alors que la seconde conduit à réifier la place de Montréal comme centre de production de grandes licences mondiales. Il convient de rappeler que le jeu vidéo est un produit globalisé et que le cas de Montréal apporte un éclairage sur les enjeux mondiaux face au médium.

Enfin, nous espérons que cette étude a suffisamment souligné l'intérêt de s'intéresser à la création, mais aussi à la production des jeux vidéo en incorporant le témoignage des développeurs eux-mêmes pour comprendre la façon dont s'actualise et s'actualisera le jeu vidéo dans les années à venir.

\section{BIBLIOGRAPHIE}

ALLIANCE NUMÉRIQUE. (2014), « Perpétuons le miracle québécois : l'avenir de l'industrie du jeu vidéo au Québec, mémoire de l'Alliance numérique déposé à la Commission d'examen sur la fiscalité québécoise ", Alliancenumerique.com, en ligne : http://alliancenumerique.com/wpcontent/uploads/2014/10/M\%C3\%A9moire_Alliance_num\%C3\%A9rique.pdf

CAILLOIS R. (1967), Les jeux et les hommes [1957], Paris, Gallimard.

CHIAPELLO L. (2014), « Évolution du rôle de designer de jeux vidéo : remise en question à l'ère du casual », Symposium annuel Histoire du jeu - Histoire culturelle du jeu vidéo / Cultural History of Video Games, présentation datant du 28 juin 2014.

COHENDET P., GRANDADAM D \& SIMON L. (2010), « The Anatomy of Creative City », Industry and innovation, vol. 17, $\mathrm{n}^{\circ} .1, \mathrm{pp} .91-111$.

ENTERTAINMENT SOFTWARE ASSOCIATION. (2014), « Essential Facts About the Canadian Video Game Industry ", Theesa.ca, en ligne :

http://theesa.ca/wp-content/uploads/2014/11/ESAC-Essential-Facts-2014.pdf 
ENTERTAINMENT SOFTWARE ASSOCIATION. (2013), « Canada's Video Game Industry in 2013, Final Report ", Theesa.ca, en ligne : http://theesa.ca/wp-content/uploads/2013/10/ESAC-VideoGames-Profile-2013-FINAL-2013-10-21-CIRC.pdf

ENTERTAINMENT SOFTWARE ASSOCIATION. (2011), « Canada's Entertainment Software Industry in 2011, a Report Prepared for the Entertainment Software Association of Canada », Theesa.ca, en ligne :

http://theesa.ca/wp-content/uploads/2011/08/SECOR_ESAC_report_eng_2011.pdf

FOUCAULT M. (1971), L'ordre du discours, Paris, Gallimard.

GRANDADAM D, COHENDET P \& SIMON L. (2013), « Places, Spaces and the Dynamics of Creativity : the Video Game Industry in Montreal », Regional studies, vol. 47, nº10, pp. 1701-1714.

HOCK-KOON S. (2011), « Expliciter les connaissances du game designer pour mieux comprendre le jeu vidéo », dans RUFAT S \& TER MINASSIAN H (dir) (2011), Les jeux vidéo comme objet de recherche, Paris, Questions théoriques, coll. Let Print., pp. 14-28.

KLINE S, DYER-WITHEFORD N \& DE PEUTER H. (2003), Digital Play: Interaction of Technology, Culture and Marketing, Montréal \& Kingston, McGill-Queen's University Press.

MARCUS G. (2002), « Au-delà de Malinowski et après Writing Culture : à propos du futur de l'anthropologie culturelle et du malaise de l'ethnographie », Ethnographiques.org, en ligne : http:// www.ethnographiques.org/2002/Marcus

OUIMET L-P. (2013), « Les petits studios de jeux vidéo se sentent négligés par Québec », Ici RadioCanada, 27 octobre 2013, en ligne : http://www.radio-canada.ca/nouvelles/Economie/ 2013/10/27/002-studios-independants-jeux-video-se-sentent-negliges-par-quebec-veulent-leurpart-du-gateau.shtml

PAILLÉ P \& MUCCHIELLI A. (2012), L'analyse qualitative en sciences humaines et sociales, Paris, Armand Collin, Collection U.

PINEAULT Y. (2014), Créer ou produire un jeu vidéo ? Étude ethnographique d'un milieu de production vidéoludique montréalais, mémoire de maîtrise, Université de Montréal, en ligne : https:// papyrus.bib.umontreal.ca/xmlui/bitstream/handle/1866/11652/

Pineault_Yann_2014_memoire.pdf ?sequence =5

RAYNA, T. \& STRIUKOVA L. (2014), « "Few to Many” : Change of Business Model Paradigm in the Video Game Industry » Communications \& Strategies, vol. 94, n², pp. 61-81.

SIMON L. (2002), Le management en univers ludique : jouer et travailler chez Ubi Soft, une entreprise du multimédia à Montréal (1998-1999).Thèse de doctorat, École des Hautes Études Commerciales Montréal, en ligne : http://www.irec.net/upload/File/memoires_et_theses/418.pdf

SIMON L \& COHENDET P. (2007), « Playing Across the Playground : Paradoxes of Knowledge Creation in the Video Game Firm », Journal of organization behavior, vol. 28, pp. 587-605.

TECHNOCOMPÉTENCES. (2013), « L'emploi dans l'industrie du jeu électronique en 2012 », TECHNOcompétences.com, en ligne : http://www.technocompetences.qc.ca/Etudes/ Etude_jeu_2012.pdf

TSCHANG T.F. (2007), « Balancing the Tensions Between Rationalization and Creativity in the Video Games Industry », Organization Science, vol. 18, nº, pp. 989-1005.

ULRICH P. (2014), « Le test de colision », Symposium annuel Histoire du jeu - Histoire culturelle du jeu vidéo / Cultural History of Video Games, présentation le 28 juin 2014. 
ZABBAN V. (2012), « Retour sur les game studies. Comprendre et dépasser les approches formelles et culturelles du jeu vidéo. » Réseaux, vol. 3, n¹73-174, pp. 137-176.

\section{NOTES}

1. Le triple A (ou AAA) est une étiquette qu'on donne à certains jeux qui se classent parmi les meilleurs ventes de l'industrie un peu comme le blockbuster du côté cinématographique. Ces productions disposent des plus grands budgets de l'industrie, de même que les plus grandes campagnes marketing; les attentes vont de pair, pour avoir un jeu respectant certains standards élevés. L'importance de ce format vidéoludique est d'autant plus important qu'à Montréal c'est autour de grandes productions que l'industrie s'est consolidée.

2. Dans une perspective ethnographique, nous emprunterons la définition partagée par tous nos informateurs de l'innovation dans le jeu vidéo, c'est-à-dire un usage différent des technologies et ressources disponibles dans le but d'offrir de nouvelles expériences vidéoludiques.

3. En tout, seize entrevues formelles ont été réalisées, cumulant près de seize heures d'enregistrement. Onze entrevues ont été effectuées avec des développeurs dans leur milieu de travail et cinq autres concernent des personnes connexes à l'industrie du jeu vidéo (deux professeurs, deux chercheurs étant aussi enseignants, et un consultant spécialiste international de l'implantation d'entreprises vidéoludiques).

4. Plusieurs de nos informateurs étaient anglophones, nous avons donc traduit leurs propos en français.

5. Pensons notamment au centre NAD, offrant une formation en animation 3D et design numérique spécialisée en jeux vidéo, à plusieurs départements d'informatiques dans des cégeps et universités, offrant des spécialisations en programmation appliquée dans le jeu vidéo ou aux récents programmes de design de jeux vidéo à l'Université de Montréal et à l'Université du Québec en Abitibi-Témiscamingue. Ces programmes sont nés de l'industrie du jeu vidéo grandissante en sol québécois et contribuent à former une main-d'œuvre qualifiée et prête à l'embauche.

6. Il s'agit d'un système de jeu se « portant » comme un masque développé Occulus VR qui devrait voir le jour en 2015.

7. Wright est cofondateur de la compagnie Maxis, à la base des séries Simcity (1993) et The Sims (2000). Meier est cofondateur des studios MicroProse et Firaxis Games, à l'origine de la série Civilization (1991). Enfin, Garriott est le créateur de la série de jeu Ultima (1981) et co-fondateur du studio Origin Systems.

8. Symposium annuel Histoire du jeu - Histoire culturelle du jeu video / Cultural History of Video Games qui a eu lieu à Montréal le 28 juin 2014 (http://www.banq.qc.ca/activites/colloque/2014/ histoire_jeu.html)

9. Premier jeu vidéo dit « de palette » à être distribué à grand tirage par Atari en 1972 et qui fut l'objet de multiples reproductions.

10. Témoignage tiré de l'émission La sphère à la radio d'Ici Radio-Canada du 24 janvier 2015 à http://ici.radio-canada.ca/emissions/la_sphere/2014-2015/ (page consultée le 3 juillet 2015)

11. Les salaires moyens dans l'Industrie du jeu vidéo et autres données connexes sont disponibles à http://www.metiers-quebec.org/portraits/graphique3.htm

12. Call of Duty est une série de jeux produit par différents studios, mais édité par Activision. Grand Theft Auto est aussi un jeu sérialisé dont les derniers titres sont produits et édités par Rockstar Games.

13. Nous avons choisi de mettre de côté le jeu mobile (ou plus largement le casual game) dans ce texte puisque notre échantillon se situait dans l'industrie du jeu triple A. L'opposition ici soulignée est celle du triple A et du jeu indépendant, mais on pourrait aussi retrouver une telle 
opposition dans le monde du casual game, où les modèles d'affaires comme la taille des entreprises divergent grandement.

14. À ce sujet, voir l'article " Jeux vidéo : alliance inédite des studios indépendants " d'Audrey Neveu et François Joly publié dans La Presse au : http://affaires.lapresse.ca/economie/quebec/ 201506/02/01-4874506-jeux-video-alliance-inedite-des-studios-independants.php （page consultée le 3 juillet 2015)

15. À titre d'exemples, pensons aux jeux Pong (1972), Tetris (1984) ou Doom (1993) qui ont tous les trois engendré une série de clones, en copiant leurs mécaniques de jeux qui étaient novatrices à leurs époques respectives.

16. À cet effet, nous avons rédigé une courte entrée de blog sur la question, disponible au : http://ypineault.com/blog/2014/10/28/industry-as-an-ecosystem-the-problems-of-a-

functionalist-analogy/

17. Il est intéressant de considérer la présentation du secteur par Investissement Québec, organisme subventionnaire gouvernemental pour les entreprises locales et étrangères : http:// www.investquebec.com/international/fr/secteurs-activite-economique/multimedia/l-explosionde-l-univers-du-jeu-video.html (page consultée le 7 juillet 2015)

\section{RÉSUMÉS}

Le jeu vidéo est un médium relativement jeune dont l'évolution s'avère incertaine. Cet article met en relief une tension qui s'exerce entre les sphères créative et productive entourant le développement d'un jeu vidéo. Nous verrons que les témoignages des développeurs et d'autres acteurs connexes de l'industrie du jeu vidéo montréalaise attestent d'une négociation constante entre ces deux espaces, où l'identité créative du développeur se voit remise en question par l'espace régulé qu'est celui du studio de production triple A. Ces témoignages seront par la suite mis en perspective face à cette même industrie qui, d'une part, semble se cristalliser autour d'une même équation du succès, mais qui de l'autre, atteste d'une diversification des modèles d'affaires, notamment avec un intérêt grandissant pour le jeu indépendant. Ce texte tente donc de comprendre les principes autour desquels s'articule l'évolution, mais aussi l'innovation du jeu vidéo en prenant le cas de l'industrie montréalaise.

The video game is a relatively young medium whose evolution is unpredictable. This article highlights a tension between the creation and production aspects in the development of a video game. We will argue that developers' and other related actors' perspectives attest of a constant negotiation between those two aspects. Consequently, the developers' creative identity is altered by the triple A regulated and production-centered space. Thereafter, we will put these testimonies into perspective in light of Montreal's video game industry which, on the one hand, seems to crystalize around its success formula but, on the other hand, shows increasingly diversified business models. We will particularly focus on independent video games. The article tries to understand the aspects surrounding the video game evolution and innovation by taking Montreal's industry as an example. 
INDEX

Mots-clés : industrie du jeu vidéo, Montréal, développeurs de jeux, ethnographie, production, création, innovation

Keywords : video game industry, Montreal, game developers, ethnography, production, creation, innovation

\section{AUTEUR}

YANN PINEAULT

Université de Montréal 